\title{
Contourlets and Sparse Image Expansions
}

\author{
Minh N. Do \\ Department of Electrical and Computer Engineering \\ University of Illinois, Urbana IL 61801
}

\begin{abstract}
Recently, the contourlet transform ${ }^{1}$ has been developed as a true two-dimensional representation that can capture the geometrical structure in pictorial information. Unlike other transforms that were initially constructed in the continuous-domain and then discretized for sampled data, the contourlet construction starts from the discrete-domain using filter banks, and then convergences to a continuous-domain expansion via a multiresolution analysis framework. In this paper we study the approximation behavior of the contourlet expansion for two-dimensional piecewise smooth functions resembling natural images. Inspired by the vanishing moment property which is the key for the good approximation behavior of wavelets, we introduce the directional vanishing moment condition for contourlets. We show that with anisotropic scaling and sufficient directional vanishing moments, contourlets essentially achieve the optimal approximation rate, $O\left((\log M)^{3} M^{-2}\right)$ square error with a best $M$-term approximation, for 2-D piecewise smooth functions with $C^{2}$ contours. Finally, we show some numerical experiments demonstrating the potential of contourlets in several image processing applications.
\end{abstract}

\section{INTRODUCTION}

Efficient representation of visual information lies at the heart of many image processing tasks, including compression, filtering, and feature extraction. Efficiency of a representation refers to the ability to capture significant information about an object of interest using a sparse description. For image compression or content-based image retrieval, the use of efficient representation implies the compactness of the compressed file or the index entry for each image in the database. For practical applications, such an efficient representation has to be obtained by structured transforms and fast algorithms.

For one-dimensional piecewise smooth signals, like scan-lines of an image, wavelets have been established as the right tool, because they provide an optimal approximation for these signals in a certain sense. ${ }^{2,3}$ In addition, the wavelet representation is amenable to efficient algorithms, in particular fast transforms and effective tree data structures. These are the key reasons for the success of wavelets in many signal processing and communication applications; for example, the wavelet transform was adopted as the transform for the new image-compression standard, JPEG-2000. ${ }^{4}$

However, natural images are not simply stacks of 1-D piecewise smooth scan-lines; discontinuity points (i.e. edges) are typically positioned along smooth curves (i.e. contours) owing to smooth boundaries of physical objects. As a result of a separable extension from 1-D bases, wavelets in 2-D are good at isolating the discontinuities at edge points, but will not see the smoothness along the contours. In addition, separable wavelets can capture only limited directional information - an important and unique feature of multidimensional signals.

These disappointing behaviors of wavelets have led to a number of new constructions of "true" two-dimensional representation that can capture the geometrical structure in visual information. In particular, the curvelet construction $^{5,6}$ of Candès and Donoho with its optimal approximation property for 2-D piecewise smooth functions with singularities on $C^{2}$ curves has shown the possibility of efficient, fixed expansions in $\mathbb{R}^{2}$. Inspired by the success of curvelets, Do and Vetterli pursued the same goal with a different point of view. Unlike other transforms that were initially constructed in the continuous-domain and then discretized for sampled data, our approach starts with a discrete-domain construction that is amenable to efficient algorithms, and then investigates its convergence to an expansion in the continuous-domain. This has resulted in the contourlet construction ${ }^{1}$ which is based on 2-D nonseparable filter banks and a directional multiresolution analysis framework.

Further author information: Email: minhdo@uiuc.edu; Web: http://www.ifp.uiuc.edu/ minhdo 
The main goal of this paper is to provide a theoretical analysis of the approximation property of the contourlet expansion. After a brief introduction to the contourlet transform, we will concentrate on its approximation behavior for 2-D functions that are smooth everywhere except discontinuities along a smooth curve. These functions model typical natural images with smooth contours. Inspired by the vanishing moment condition which is the key for the good approximation property of wavelets for 1-D piecewise smooth functions, we introduce the directional vanishing moment condition for the good approximation property of contourlets. We show that with the anisotropy scaling relation and sufficient directional vanishing moments, contourlets provide an optimal approximation decay for the 2-D piecewise smooth functions with $C^{2}$ contours. Finally, we will show some numerical results to demonstrate the potential of contourlets in several image processing applications.

\section{THE CONTOURLET CONSTRUCTION}

\subsection{Discrete-Domain: A Filter Bank Approach}

One way to obtain a sparse expansion for images with smooth contours is first to apply a multiscale, waveletlike transform to capture the edge points; and then use a local directional transform to gather the nearby edge points into contour segments. This prompted us ${ }^{1}$ to construct a multiscale and directional filter bank, where the Laplacian pyramid $(\mathrm{LP})^{7}$ is first used to capture the point discontinuities, then followed by a directional filter bank $(\mathrm{DFB})^{8}$ to link point discontinuities into linear structures. The overall result is an image expansion using basic elements like contour segments, and thus are named contourlets. The block structure for the contourlet filter bank is shown in Figure 1 together with an example of its frequency partition.

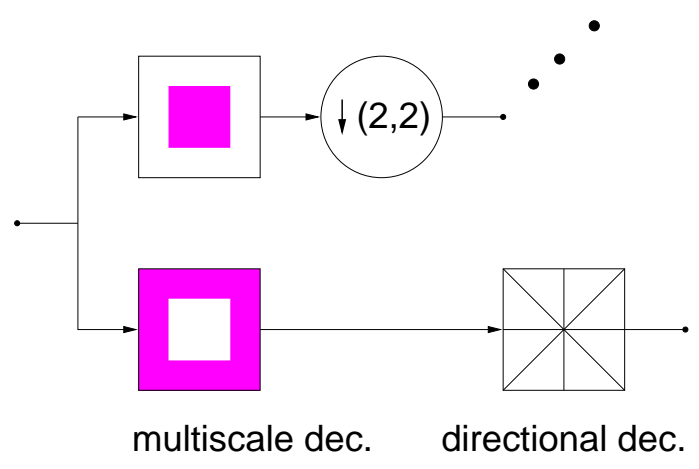

(a)

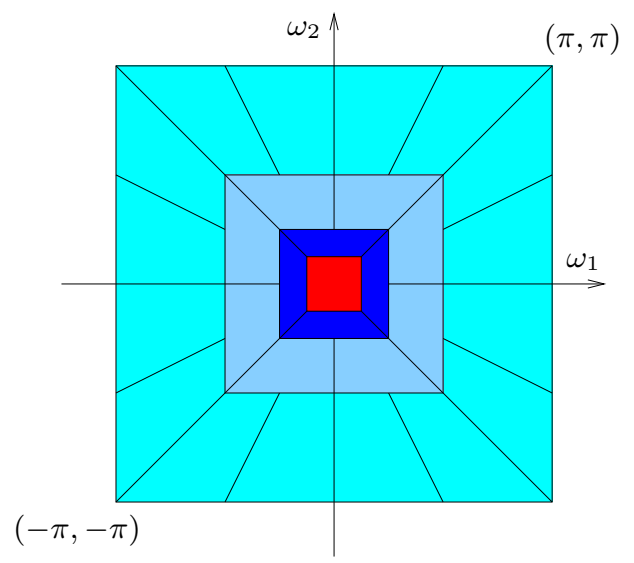

(b)

Figure 1. (a) Block diagram of the contourlet filter bank. First, a standard multiscale decomposition into octave bands is computed, where the lowpass channel is subsampled and iterated, while a directional filter bank is applied the to the bandpass channel. (b) Resulting frequency division, where the number of directions is increased with frequency

With perfect reconstruction LP and DFB, the contourlet filter bank achieves perfect reconstruction, and thus it is a frame operator for 2-D signals. With orthogonal filters, the contourlet transform was shown ${ }^{1}$ to be a tight frame. The contourlet filter bank has the same redundancy as the LP - up to $33 \%$ when subsampling by two in each dimension. Since the implementation of both the LP and the DFB are based on iterated filter banks, when the numbers of decomposition levels in each iterated filter bank is finite, it is can be checked that each contourlet coefficient require $O(1)$ operations. Thus the computational complexity of the contourlet transform is $O(N)$ for an image of $N$ pixels.

\subsection{Continuous-Domain: Convergence via Multiresolution Analysis}

Similar to the wavelet filter bank,,$^{3,9}$ the contourlet filter bank has an associated continuous-domain expansion in $L_{2}\left(\mathbb{R}^{2}\right)$ that is connected via a directional multiresolution analysis framework. ${ }^{1}$ With orthogonal filters, 

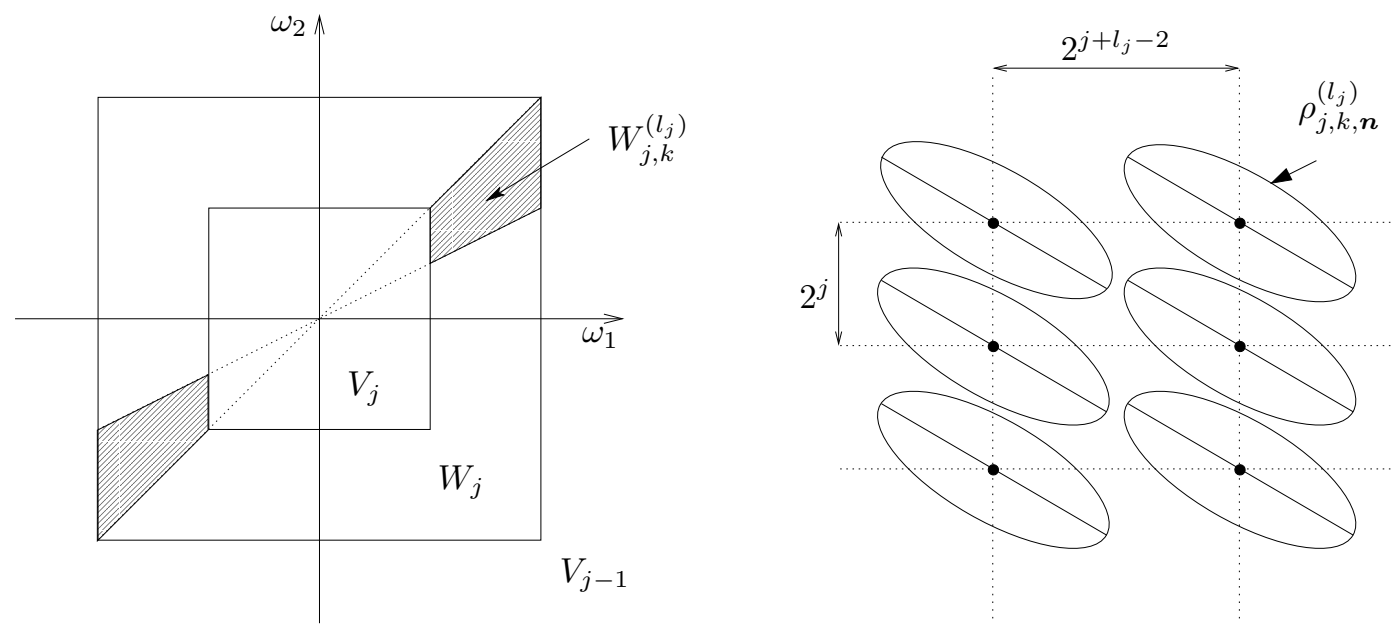

Figure 2. (a) Multiscale and multidirectional subspaces generated by the contourlet transform which is illustrated on a 2-D spectrum decomposition. (b) Sampling grid of a "mostly horizontal" subspace $W_{j, k}^{l_{j}}$. For "mostly vertical" subspaces, the grid is transposed.

through its iterated filter bank, the continuous-domain contourlet transform decomposes $L_{2}\left(\mathbb{R}^{2}\right)$ into multiscale and multidirectional subspaces (see Figure 2(a))

$$
L_{2}\left(\mathbb{R}^{2}\right)=V_{j_{0}} \oplus\left(\bigoplus_{j \leq j_{0}}\left(\bigoplus_{k=0}^{2^{l_{j}}} W_{j, k}^{\left(l_{j}\right)}\right)\right)
$$

where the familiar wavelet detail subspace $W_{j}$ at scale $2^{j}$ is decomposed into $2^{l_{j}}$ directional subspaces $W_{j, k}^{\left(l_{j}\right)}$. The approximation subspace $V_{j_{0}}$ is spanned by an orthogonal basis of a scaling function and its translation $\left\{\phi_{j_{0}, \boldsymbol{n}}(\boldsymbol{t})=\phi_{j_{0}}\left(\boldsymbol{t}-2^{j} \boldsymbol{n}\right)\right\}_{\boldsymbol{n} \in \mathbb{Z}^{2}}$. Each subspace $W_{j, k}^{\left(l_{j}\right)}$ is spanned by a tight frame of a translated family of contourlet functions

$$
\left\{\rho_{j, k, \boldsymbol{n}}^{\left(l_{j}\right)}(\boldsymbol{t})=\rho_{j, k}^{\left(l_{j}\right)}\left(\boldsymbol{t}-2^{j-1} \boldsymbol{S}_{k}^{\left(l_{j}\right)} \boldsymbol{n}\right)\right\}_{\boldsymbol{n} \in \mathbb{Z}^{2}}
$$

where $\boldsymbol{S}_{k}^{\left(l_{j}\right)}=\operatorname{diag}\left(2^{l_{j}-1}, 2\right)$ for $0 \leq k<2^{l_{j}-1}$ (the mostly horizontal directions) and $\boldsymbol{S}_{k}^{\left(l_{j}\right)}=\operatorname{diag}\left(2,2^{l_{j}-1}\right)$ for $2^{l_{j}-1} \leq k<2^{l_{j}}$ (the mostly vertical directions). This means that the subspace $W_{j, k}^{(l)}$ is defined on a rectangular grid with intervals $2^{j+l-2} \times 2^{j}$ or $2^{j} \times 2^{j+l-2}$, depending on whether it is mostly horizontal or vertical (see Figure 2(b)).

Combining all scales, directions and locations, we have that for a sequence of finite positive integers $\left\{l_{j}\right\}_{j \leq j_{0}}$, the family

$$
\left\{\phi_{j_{0}, \boldsymbol{n}}, \rho_{j, k, \boldsymbol{n}}^{\left(l_{j}\right)}(\boldsymbol{t})\right\}_{j \leq j_{0}, 0 \leq k \leq 2^{l_{j}}-1, \boldsymbol{n} \in \mathbb{Z}^{2}}
$$

is a tight frame for $L_{2}\left(\mathbb{R}^{2}\right)$. The indexes $j, k$, and $\boldsymbol{n}$ specify the scale, direction and, location, respectively. The contourlet frame in (2) the following distinguishing features:

1. The contourlet expansions are defined on rectangular grids, and thus offer a seamless translation to the discrete world, where image pixels are stored on a rectangular grid. To achieve this "digital-friendly" feature, the contourlet kernel functions $\rho_{j, k}^{\left(l_{j}\right)}$ have to be different for different directions $k$ and cannot be obtained by simply rotating a single function. This is a key difference between the contourlet and the curvelet $^{5,6}$ systems. 
2. It is easy to verify that with FIR filters, the iterated contourlet filter bank leads to compactly supported contourlet frames. More precisely, the contourlet function $\rho_{j, k, \boldsymbol{n}}^{\left(l_{j}\right)}$ has support of size width $\approx W 2^{j}$ and length $\approx L 2^{j+l_{j}-2}$. In other words, at each scale and direction, the set $\left\{\rho_{j, k, \boldsymbol{n}}^{\left(l_{j}\right)}\right\}_{\boldsymbol{n} \in \mathbb{Z}^{2}}$ tiles the plane $\mathbb{R}^{2}$. As a result, to make the contourlet expansion satisfy the anisotropy scaling law like curvelets width $\approx l e n g t h^{2},{ }^{5}$ we simply set

$$
l_{j}=l_{j_{0}}-\left\lfloor\left(j-j_{0}\right) / 2\right\rfloor, \quad \text { for } j \leq j_{0} .
$$

This means that the number of directions is doubled at every other finer scale in the contourlet pyramid.

3. Since the contourlet functions are defined via the iterated filter banks; like wavelets, the contourlet expansion has fas filter bank algorithms and convenient tree structures.

\section{CONTOURLET APPROXIMATION}

\subsection{Directional vanishing moment}

The proposed 2-D filter bank and its associated continuous-domain frames in the previous sections provide a framework for constructing general contourlet expansions. Since our goal is to develop sparse expansions for images having smooth contours, the next important issues are: (1) what conditions should we impose in order to obtain a sparse contourlet expansion for certain classes of images; and (2) how can we design filter banks that can lead to contourlet expansions satisfying those conditions. We will consider the first issue here while the second one will be addressed in a forthcoming paper.

In analogy with the wavelet case, while most two-channel filter banks would lead to discrete and continuous wavelet bases, not all guarantee sparse expansions. Wavelet approximation theory brought a new condition into filter bank design, which earlier only focused on designing filters with good frequency selection properties. This new condition requires wavelet function to have a sufficient number of vanishing moments or equivalently the lowpass filter must have enough "zeros at $\omega=\pi$ ". The vanishing-moments property is the key for the sparse expansion of piecewise smooth signals by wavelets. ${ }^{3,10,11}$ Intuitively, wavelets with vanishing moments are orthogonal with polynomial signals, and thus only a few wavelet basis functions around discontinuity points would "feel" these singularities and lead to significant coefficients. ${ }^{12}$

In the contourlet case, our target for approximation are 2-D piecewise smooth functions with discontinuities along smooth curves. For this type of signals, the singularities are localized in both space and direction. More specifically, a local region around a smooth contour has a 2-D singularity that can be approximated by straight line separating two polynomial surfaces. Since each contourlet function $\rho_{j, k, \boldsymbol{n}}^{\left(l_{j}\right)}$ is localized at a specific scale, direction and location, it is desirable that only the contourlet basis functions which are located and oriented around the discontinuity line would "feel" this singularity. This requirement can be satisfied if all 1-D slices in a certain direction of a contourlet kernel function $\rho_{j, k}^{\left(l_{j}\right)}$ have vanishing moments. We refer to such a contourlet function as having directional vanishing moments (DVMs).

Definition 3.1 (Directional VAnishing moment). A 2-D function $\rho\left(t_{1}, t_{2}\right)$ is said to have L-order directional vanishing moment along a direction $\boldsymbol{u}=\left(u_{1}, u_{2}\right)^{T}$ if, depending on whether $u_{1} \neq 0$ or $u_{2} \neq 0$, all 1-D slices of that function along direction $\boldsymbol{u}$

$$
\begin{aligned}
& \rho_{\boldsymbol{u}, d}^{(1)}(t)=\rho\left(t, t u_{2} / u_{1}-d\right), \quad \text { or } \\
& \rho_{\boldsymbol{u}, d}^{(2)}(t)=\rho\left(t u_{1} / u_{2}-d, t\right),
\end{aligned}
$$

have $L$ vanishing moments. That means

$$
\int_{-\infty}^{\infty} \rho_{\boldsymbol{u}, d}^{(i)}(t) t^{n}=0, \quad \forall d \in \mathbb{R}, 0 \leq n<L
$$

for either $i=1$ or $i=2 .^{*}$

\footnotetext{
*If both $u_{1} \neq 0$ or $u_{2} \neq 0$ then it is easy to see that the two conditions for $i=1$ and $i=2$ are equivalent.
} 
For a contourlet functions $\rho_{j, k}^{(l)}(\boldsymbol{t})$ constructed from filter banks, the DVM property can be enforced via filter bank design as in the wavelet case. We observe that the DVM property also holds in other 2-D expansions. In particular, 2-D separable wavelets have directional vanishing moments in the horizontal and vertical directions, which make wavelets especially good in capturing horizontal and vertical edges. Ridgelets, ${ }^{13}$ which offer an optimal representation for 2-D functions that are smooth away from a discontinuity along a line, have directional vanishing moments in all but one direction.

\subsection{Intuition Behind the Proof}

Now we show that a contourlet expansion that satisfies the anisotropy scaling law and has sufficient DVMs achieves the optimal non-linear approximation behavior for 2-D piecewise smooth functions with discontinuities along smooth curves. We first present a heuristic argument. Consider a simple piecewise smooth function $f=\mathbf{1}_{\Omega}$ defined on the unit square $[0,1]^{2}$ with "black" and "white" regions separated by a discontinuity curve $\delta \Omega$, which is twice differentiable and has finite length.

Recall that for a contourlet frame (2) to satisfy the anisotropy scaling condition, $l_{j}$ has to follow (3). For simplicity, set $j_{0}=0$ and $l_{0}=2 .^{\dagger}$ This leads to a contourlet frame which at scale $2^{j}(j=-1,-2, \ldots)$ has about $2^{-j / 2}$ directions and each contourlet function $\rho_{j, k, n}$ has support size of order width $\sim 2^{j}$ and length $\sim 2^{j / 2}$. It follows that the maximum amplitude of $\rho_{j, k, n}$ is of order of $A_{j} \sim 2^{-3 j / 4}$.

Suppose that the contourlet kernel functions $\rho_{j, k}$ have one-order DVMs along a dense enough set of directions (this will be made precise later). Then we can localize the support area of $\rho_{j, k, \boldsymbol{n}}$ that would be effected by the discontinuity curve $\delta \Omega$ to be "sandwiched" between two lines parallel to the tangent of the curve (see Figure 3(b)). Outside that area, because of the vanishing moments on the tangent direction, the product $f \cdot \rho_{j, k, n}$ has zero line integrals along that direction. Denote $d_{j, k, \boldsymbol{n}}$ to be the length of $\delta \Omega$ that intersects with the support of $\rho_{j, k, \boldsymbol{n}}$. Because the curve is twice differentiable, using Taylor expansion, the width of the affected area is of the order $d_{j, k, n}^{2}$. Thus, the corresponding contourlet coefficient behaves like

$$
\left|\left\langle f, \rho_{j, k, \boldsymbol{n}}\right\rangle\right| \sim A_{j} \cdot d_{j, k, \boldsymbol{n}}^{3} \sim 2^{-3 j / 4} \cdot d_{j, k, \boldsymbol{n}}^{3} .
$$

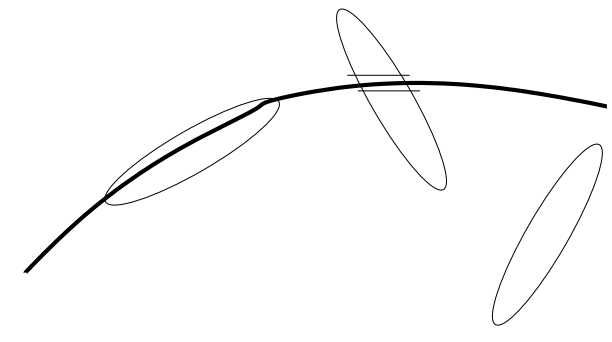

(a)

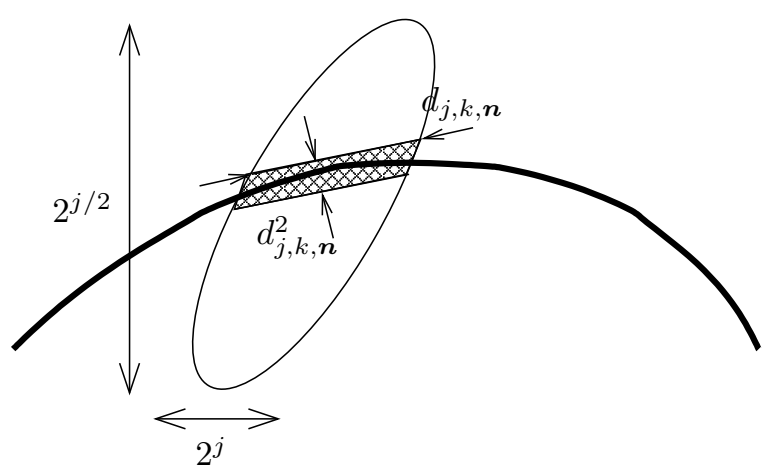

(b)

Figure 3. (a) Contourlet functions correspond to three types of coefficients: (from left to right) significant, insignificant, and zero. (b) A zoom-in examination of a contourlet basis function that intersects with a discontinuity curve.

At a scale $2^{j}$, the contourlet coefficients $\left\langle f, \rho_{j, k, n}\right\rangle$ can be classified into three types (see Figure 3(a)). The first type contains significant contourlet coefficients, which correspond to the contourlet basis functions $\rho_{j, k, n}$ whose support is aligned with the discontinuity curve $\delta \Omega$. For these contourlet functions, we have $d_{j, k, n} \sim 2^{j / 2}$

\footnotetext{
${ }^{\dagger}$ Other values of $l_{0}$ only changes the constant but not the asymptotic approximation rate.
} 
and from (4), the significant contourlet coefficients behave as $\left|\left\langle f, \rho_{j, k, \boldsymbol{n}}\right\rangle\right| \sim 2^{-3 j / 4}\left(2^{j / 2}\right)^{3}=2^{3 j / 4}$. In addition, because the curve is finite length, there are $O\left(2^{-j / 2}\right)$ of them.

The second type contains insignificant contourlet coefficients, which correspond to contourlet functions $\rho_{j, k, n}$ whose supports intersect with the discontinuity curve but the direction does not match. From Figure 3(b) we see that for most of these contourlet functions $d_{j, k, n} \sim 2^{j}$, and using (4) the insignificant contourlet coefficients behave as $\left|\left\langle f, \rho_{j, k, \boldsymbol{n}}\right\rangle\right| \sim 2^{-3 j / 4}\left(2^{j}\right)^{3}=2^{9 j / 4}$. In addition, most of the directions (except the ones that match with the discontinuity curve) fall into this category, and thus we have an order of $2^{-j / 2} 2^{-j}=2^{-3 j / 2}$ insignificant contourlet coefficients. The final type contains zero contourlet coefficients which correspond to contourlet functions $\rho_{j, k, n}$ whose support does not intersect with the discontinuity curve $\delta \Omega$.

For a best $M$-term approximation, based on the above rates of decay of contourlet coefficients and by setting the threshold $T=2^{9 J / 4}(J \ll 0)$, we keep the significant coefficients up to level $3 J$ and insignificant coefficients up to level $J$. Then the total number of retained coefficients is:

$$
M \sim \sum_{3 J}^{0} 2^{j / 2}+\sum_{J}^{0} 2^{3 j / 2} \sim 2^{-3 J / 2} .
$$

The distortion due to truncation is:

$$
\begin{aligned}
\left\|f-\hat{f}_{M}^{\text {contourlet }}\right\|^{2} & \sim \sum_{-\infty}^{3 J-1} 2^{-j / 2}\left(2^{3 j / 4}\right)^{2}+\sum_{-\infty}^{J-1} 2^{-3 j / 2}\left(2^{9 j / 4}\right)^{2} \\
& \sim 2 \sum_{-\infty}^{J-1} 2^{3 j} \sim 2^{3 J}
\end{aligned}
$$

Combining (5) and (6) we see that the nonlinear approximation error by the contourlet expansion decays like $\left\|f-\hat{f}_{M}^{\text {contourlet }}\right\| \sim M^{-2}$. For comparison, the approximation error of the same function $f$ by wavelets decays like $M^{-1}$ (see for example ${ }^{3}$ ). Because the "complexity" of $f$ is at least equal to the "complexity" of $\delta \Omega$, which is a $C^{2}$ curve, no other approximation scheme can achieve a better rate than $M^{-2}$. In this sense, the contourlet expansion achieves the optimal approximation rate for piecewise smooth functions with $C^{2}$ contours.

\subsection{Finer Analysis}

The above heuristic argument on the approximation property of contourlets needs more precise and finer analysis on the following two points. First, how dense is the set of directions that the contourlet functions are required to have DVMs. Second, it is inaccurate to claim that all insignificant coefficients would have $d_{j, k, \boldsymbol{n}} \sim 2^{j}$. In fact, $d_{j, k, \boldsymbol{n}}$ decays from $2^{j / 2}$ to $2^{j}$ as the direction of the intersected contourlet slowly turn away from the direction of the discontinuity curve. We need to analyze this decay and its effect in the overall approximation rate.

The first point is illustrated in Figure 4(a), where we suppose that instead of having DVMs on the tangent direction of the discontinuity curve, we only have DVMs on a direction that is $\theta$ away. Then simple geometrical argument shows that instead of $d^{3}$, the affected area would be upper bounded by

$$
d^{3}+d \cdot d \tan \theta
$$

Thus, if $\tan \theta \leq A_{1} d$ then the approximation rate in the above analysis will be unchanged. To achieve this and since $d_{j, k, \boldsymbol{n}} \leq A_{2} 2^{j / 2}$, the maximum gap $\theta_{j}$ between directions that $\rho_{j, k, \boldsymbol{n}}$ has DVMs must be of the order

$$
\theta_{j} \leq A 2^{j / 2} .
$$

Recall that at scale $2^{j}$, there would be $2^{-j / 2}$ directions. Hence, the number of directions on which the contourlet function $\rho_{j, k}$ has DVMs grows with the same order as the number of directions in the directional filter bank. 

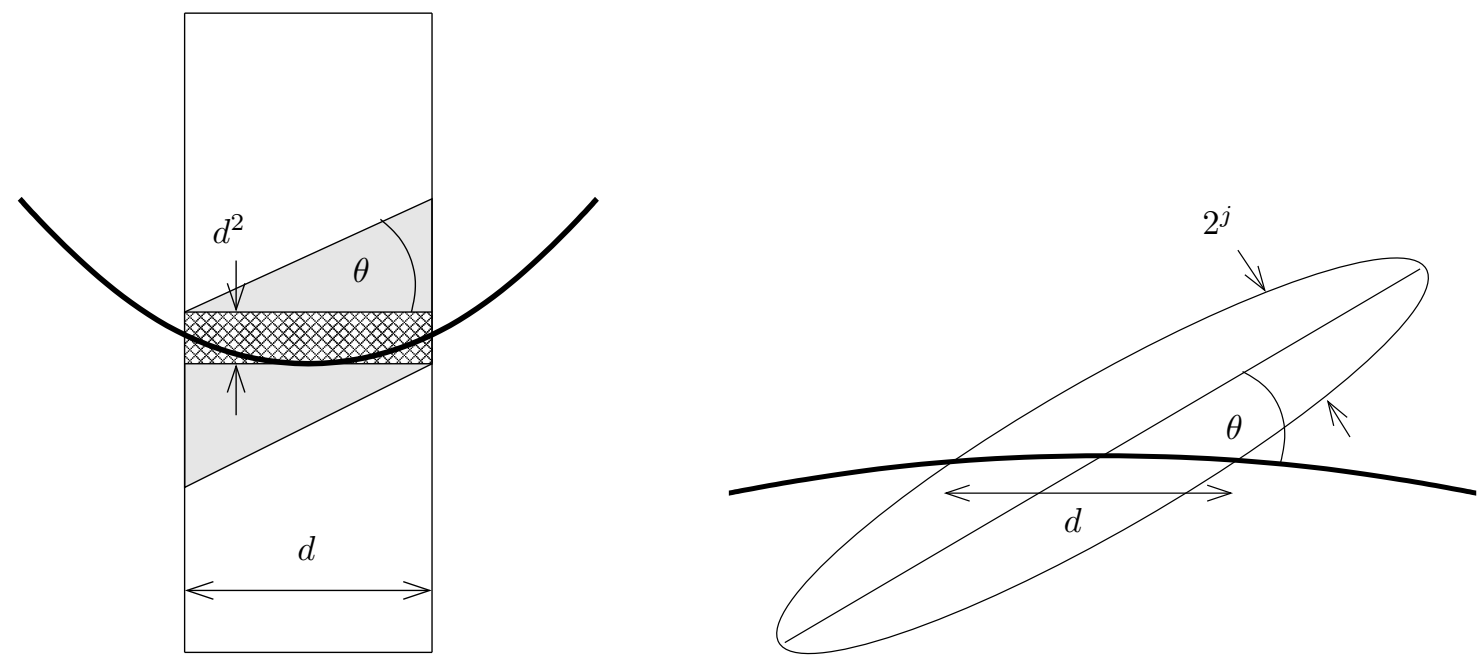

Figure 4. Finer analysis of contourlet approximation. (a) Illustrating the allowable gap in angle with directional vanishing moments. (b) Finer approximation of the intersection.

For the second point, Figure 4(b) illustrates the situation where a contourlet function $\rho_{j, k, n}$ intersects with the discontinuity curve $\delta \Omega$ and is $\theta$ angle away from the tangent direction, $0<\theta \leq \pi / 2$. In that case, using geometry we have

$$
\sin \theta \sim 2^{j} / d_{j, k, \boldsymbol{n}}, \quad \text { or } \quad d_{j, k, \boldsymbol{n}} \sim 2^{j} / \sin \theta .
$$

Since there is about $2^{-j / 2}$ directions, as $\theta$ increases almost uniformly with a step size of the order $2^{j / 2}$ to $\pi / 2$ (i.e. the contourlet function slowly turns away from the discontinuity direction), $d_{j, k, n}$ decays like $2^{j} /\left(m 2^{j / 2}\right)=2^{j / 2} m^{-1}$, for $1 \leq m \leq 2^{-j / 2}$. Correspondingly, the number of contourlets functions with these intersection lengths grows like $2^{-j / 2} m$. Using (4), it follows that the non-zero contourlet coefficients decay like $2^{-3 j / 4}\left(2^{j / 2} m^{-1}\right)^{3}=2^{3 j / 4} m^{-3}$, for $1 \leq m \leq 2^{-j / 2}$.

Now by setting the threshold $T=2^{9 J / 4}(J \ll 0)$, then at scale $2^{j}$ we would keep coefficients with $m \leq$ $2^{(j-3 J) / 4}$. This leads to the following number of retained coefficients:

$$
\begin{aligned}
M & \sim \sum_{j=J}^{0} \sum_{m=1}^{2^{-j / 2}} 2^{-j / 2} m+\sum_{j=3 J}^{J-1} \sum_{m=1}^{2^{(j-3 J) / 4}} 2^{-j / 2} m \\
& \sim \sum_{j=J}^{0} 2^{-j / 2}\left(2^{-j / 2}\right)^{2}+\sum_{j=3 J}^{J-1} 2^{-j / 2}\left(2^{(j-3 J) / 4}\right)^{2} \\
& =2^{-3 J / 2}+(-2 J) 2^{-3 J / 2} \sim(-J) 2^{-3 J / 2} .
\end{aligned}
$$

The distortion due to truncation is

$$
\begin{aligned}
\left\|f-\hat{f}_{M}^{\text {contourlet }}\right\|^{2} & \sim \sum_{j=3 J}^{J-1} \sum_{m=2^{(j-3 J) / 4+1}}^{2^{-j / 2}} 2^{-j / 2} m\left(2^{3 j / 4} m^{-3}\right)^{2}+\sum_{j=-\infty}^{3 J-1} \sum_{m=1}^{2^{-j / 2}} 2^{-j / 2} m\left(2^{3 j / 4} m^{-3}\right)^{2} \\
& \sim \sum_{j=3 J}^{J-1} 2^{j} \sum_{m=2^{(j-3 J) / 4+1}}^{2^{-j / 2}} m^{-5}+\sum_{j=-\infty}^{3 J-1} 2^{j} \sum_{m=1}^{2^{-j / 2}} m^{-5} .
\end{aligned}
$$


To simplify the last expression, we use the following approximation

$$
\sum_{m=a}^{b} m^{-5} \sim \int_{a}^{b} x^{-5} d x=\left(a^{-4}-b^{-4}\right) / 4 .
$$

Substitute this back into (9) we obtain

$$
\left\|f-\hat{f}_{M}^{\text {contourlet }}\right\|^{2} \sim(-J) 2^{3 J} .
$$

Combining (8) with (10) we have the following nonlinear approximation error by contourlets

$$
\left\|f-\hat{f}_{M}^{\text {contourlet }}\right\|^{2} \sim(-J)^{3} M^{-2} \sim(\log M)^{3} M^{-2},
$$

which except for a log factor, achieves the optimal rate as before. Interestingly, this is exactly the same approximation behavior derived for curvelets, ${ }^{6}$ where curvelets are compactly supported in frequency and the approximation analysis was carried in the Fourier domain. We summarize the above results in the following theorem.

THEOREM 3.2. Suppose that the contourlet frame (2) satisfies the anisotropy scaling law (3) and each contourlet kernel function $\rho_{j, k}$ has directional vanishing moments on a set of directions with maximum gap of $A 2^{j / 2}$. Then for a function $f$ that is $C^{2}$ smooth away from a $C^{2}$ curve, the best $M$-term approximation by this contourlet frame achieves

$$
\left\|f-\hat{f}_{M}^{\text {contourlet }}\right\|^{2} \leq C(\log M)^{3} M^{-2} .
$$

\section{NUMERICAL EXPERIMENTS}

All experiments in this section use a wavelet transform with "9-7" biorthogonal filters ${ }^{14,15}$ and 6 decomposition levels. For the contourlet transform, in the LP stage we also use the "9-7" filters. The choice of the "9-7" biorthogonal filters rather than orthogonal one is motivated by the fact that these filters are very popular and successful in image processing practice (partly because they are linear phase) and actually they are close to being orthogonal. In the DFB stage we use the "23-45" biorthogonal quincunx filters designed by Phoong et al. ${ }^{16}$ and modulated to them to obtain the biorthogonal fan filters. Apart from being linear phase and nearly orthogonal, these fan filters are close to have the ideal frequency response and thus can approximate the directional vanishing moment condition. The drawback is that they have large support which create a large number of significant coefficients near edges. As mentioned before, designing optimized contourlet filters is a topic to be studied further.

The number of DFB decomposition levels is doubled at every other finer scale and is equal to 5 at the finest scale. Note that in this case, both the wavelet and the contourlet transforms share the same detail subspaces $W_{j}$. The difference is that each detail subspace $W_{j}$ in the wavelet transform is represented by a basis with three directions, whereas in the contourlet transform it is represented by a redundant frame with many more directions. An example of the contourlet transform is shown in Figure 5.

\subsection{Non-linear approximation}

We compare the non-linear approximation (NLA) performances of the wavelet and the contourlet transforms. In these NLA experiments, for a given value $M$, we select the $M$-most significant coefficients in each transform domain, and then compare the reconstructed images from these sets of $M$ coefficients. Since the two transforms share the same detail subspaces, it is possible to restrict the comparison in these subspaces. We expect that most of the refinement happens around the image edges.

Figure 6 shows sequences of nonlinear approximated images at the finest detailed subspace $W_{j}$ using the wavelet and the contourlet transforms, respectively, for the input peppers image. The wavelet scheme is seen to 


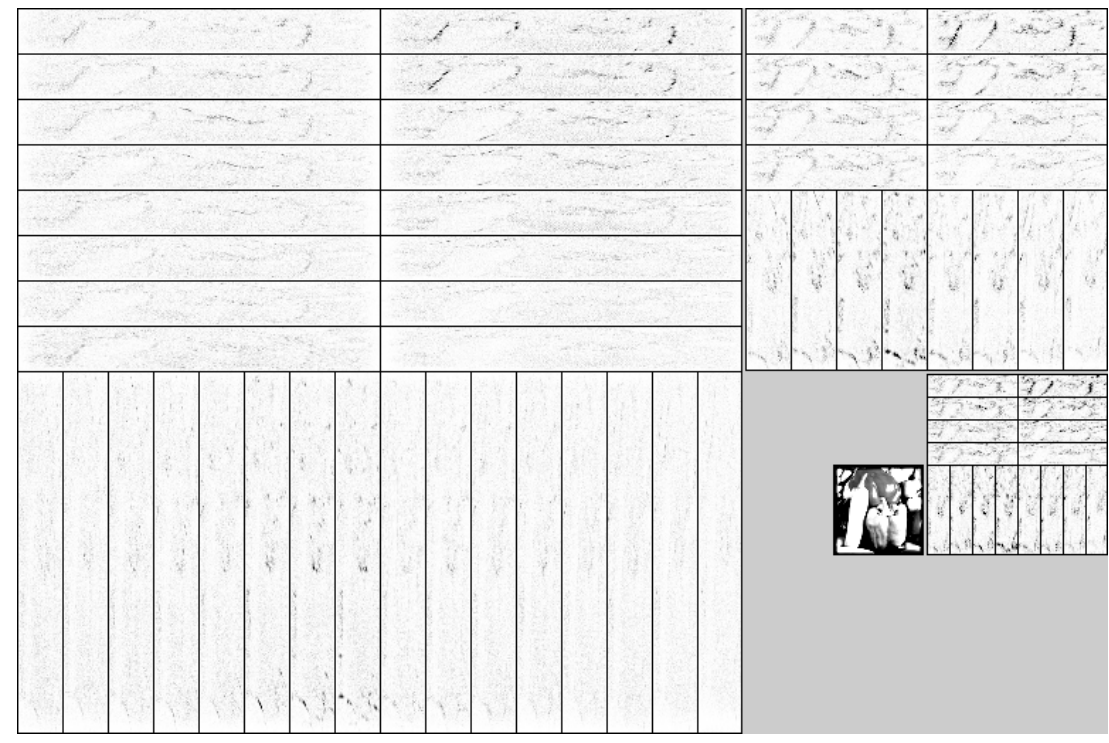

Figure 5. Example of the discrete contourlet transform, applied to the peppers image.

$M=4$
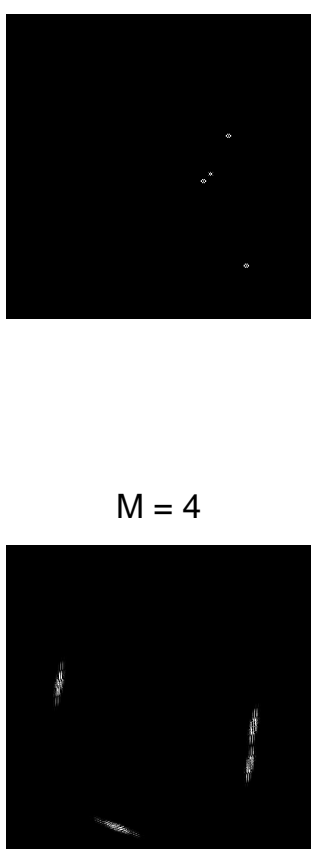

$M=16$

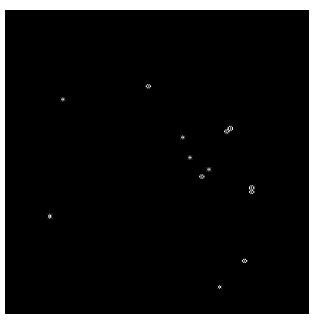

(a) Using wavelets
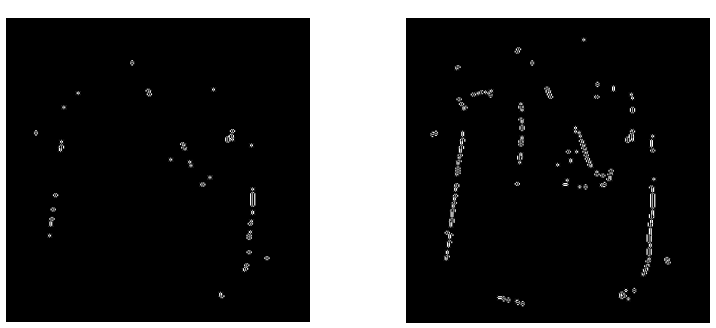

$M=16$

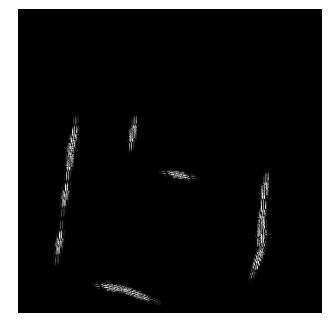

$M=64$

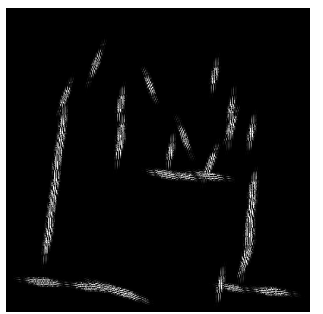

$M=256$

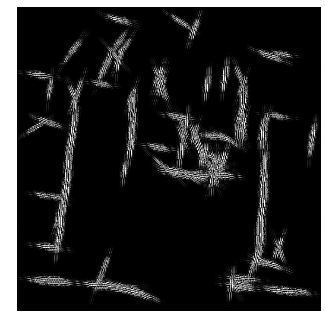

(b) Using contourlets

Figure 6. Sequence of images showing the nonlinear approximations of the peppers image using $M$ most significant coefficients at the finest detailed subspace $W_{j}$, which is shared by both the wavelet and contourlet transforms.

slowly capture contours by isolated "dots". By contrast, the contourlet scheme quickly refines by well-adapted "sketches", in much the same way as the "X-let" painter discussed in the Introduction.

Figure 7 shows a detailed comparison of two nonlinear approximated images by the wavelet and contourlet 
transforms using the same number of coefficients. Contourlets are shown to be superior compared with wavelets in capturing fine contours (directional textures on cloths). In addition, there is a significant gain of $1.46 \mathrm{~dB}$ in peak signal-to-noise ratio (PSNR) by contourlets.

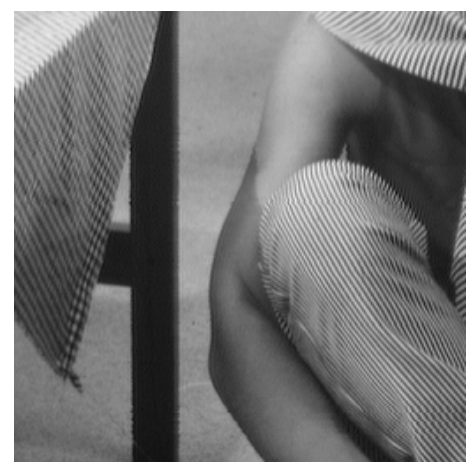

Original image

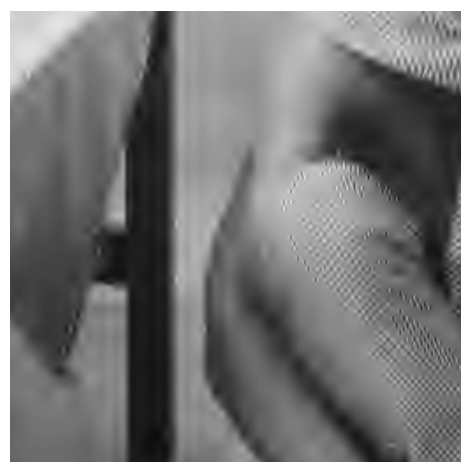

Wavelet NLA: PSNR $=24.34 \mathrm{~dB}$

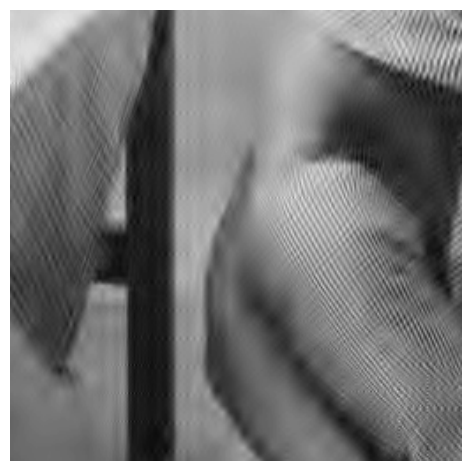

Contourlet NLA: PSNR $=25.70 \mathrm{~dB}$

Figure 7. Nonlinear approximations (NLA) by the wavelet and contourlet transforms. In each case, the original image Barbara of size $512 \times 512$ is reconstructed from the 4096-most significant coefficients. Only part of images are shown for detail comparison.

\subsection{Denoising}

The improvement in approximation by contourlets based on keeping the most significant coefficients will directly lead to improvements in applications, including compression, denoising, and feature extraction. In particular, for image denoising, random noise will generate significant wavelet coefficients just like edges, but is less likely to generate significant contourlet coefficients. Consequently, a simple thresholding scheme ${ }^{17}$ applied on the contourlet transform is more effective in removing the noise than it is for the wavelet transform.

Figure 8 displays a "zoom-in" comparison of denoising when applying wavelet and contourlet hard-thresholding on the Lena image. The contourlet transform is shown to be more effective in recovering smooth contours, both visually as well as in signal-to-noise ratio (SNR). A more sophisticated denoising scheme that takes into account the dependencies across scales, directions and locations in the contourlet domain using a hidden Markov tree model is presented in ${ }^{18}$ and shows further improvements.
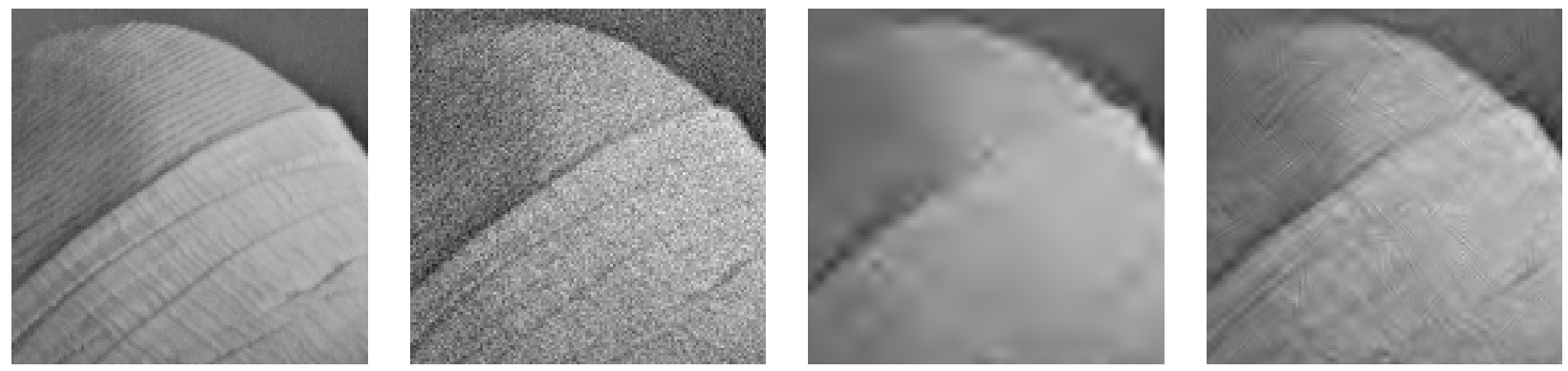

Figure 8. Denoising experiments. From left to right are: original image, noisy image (SNR $=9.55 \mathrm{~dB})$, denoising using wavelets $(\mathrm{SNR}=13.82 \mathrm{~dB})$, and denoising using contourlets $(\mathrm{SNR}=15.42 \mathrm{~dB})$.

\section{CONCLUSION}

The contourlet construction offers new directional multiresolution expansions in both discrete and continuous domains. For contourlets to provide sparse expansions for images with smooth contours, we suggest that the key property is directional vanishing moment. With anisotropic scaling and sufficient directional vanishing moments, 
we show that the contourlet expansion achieves the optimal approximation rate for piecewise smooth functions in $\mathbb{R}^{2}$ with $C^{2}$ contours. Experiments with real images indicate the potential of contourlets in several image processing applications. A key issue for future research is to design optimized contourlet filters that satisfies the directional vanishing moment condition.

Acknowledgment. The author would like to thank Prof. Martin Vetterli for fruitful interactions on directional multiresolution image representations.

\section{REFERENCES}

1. M. N. Do and M. Vetterli, "Contourlets," in Beyond Wavelets, G. V. Welland, ed., Academic Press, New York, 2003. to appear, http://www.ifp.uiuc.edu/ minhdo/publications.

2. D. L. Donoho, M. Vetterli, R. A. DeVore, and I. Daubechies, "Data compression and harmonic analysis," IEEE Trans. Inform. Th. 44, pp. 2435-2476, October 1998.

3. S. Mallat, A Wavelet Tour of Signal Processing, Academic Press, 2nd ed., 1999.

4. A. Skodras, C. Christopoulos, and T. Ebrahimi, "The JPEG 2000 still image compression standard," IEEE Signal Processing Magazine 18, pp. 36-58, Sep. 2001.

5. E. J. Candès and D. L. Donoho, "Curvelets - a suprisingly effective nonadaptive representation for objects with edges," in Curve and Surface Fitting, A. Cohen, C. Rabut, and L. L. Schumaker, eds., Vanderbilt University Press, (Saint-Malo), 1999.

6. E. J. Candès and D. L. Donoho, "New tight frames of curvelets and optimal representations of objects with smooth singularities," tech. rep., Department of Statistics, Stanford University, 2002. submitted.

7. P. J. Burt and E. H. Adelson, "The Laplacian pyramid as a compact image code," IEEE Trans. Commun. 31, pp. 532-540, April 1983.

8. R. H. Bamberger and M. J. T. Smith, "A filter bank for the directional decomposition of images: Theory and design," IEEE Trans. Signal Proc. 40, pp. 882-893, April 1992.

9. M. Vetterli and J. Kovačević, Wavelets and Subband Coding, Prentice-Hall, Englewood Cliffs, NJ, 1995.

10. I. Daubechies, Ten Lectures on Wavelets, SIAM, Philadelphia, PA, 1992.

11. G. Strang and T. Nguyen, Wavelets and Filter Banks, Wellesley Cambridge Press, Boston, 1996.

12. M. Vetterli, "Wavelets, approximation and compression," IEEE SP Mag. , pp. 59-73, Sep. 2001.

13. E. J. Candès and D. L. Donoho, "Ridgelets: a key to higher-dimensional intermittency?," Phil. Trans. R. Soc. Lond. A. , pp. 2495-2509, 1999.

14. A. Cohen, I. Daubechies, and J.-C. Feauveau, "Biorthogonal bases of compactly supported wavelets," Commun. on Pure and Appl. Math. 45, pp. 485-560, 1992.

15. M. Vetterli and C. Herley, "Wavelets and filter banks: Theory and design," IEEE Trans. Signal Proc. 40, pp. 2207-2232, September 1992.

16. S.-M. Phoong, C. W. Kim, P. P. Vaidyanathan, and R. Ansari, "A new class of two-channel biorthogonal filter banks and wavelet bases," IEEE Trans. Signal Proc. 43, pp. 649-665, Mar. 1995.

17. D. Donoho and I. Johnstone, "Ideal spatial adaptation via wavelet shrinkage," Biometrika 81, pp. 425-455, Dec. 1994.

18. D. D.-Y. Po and M. N. Do, "Directional multiscale modeling of images using the contourlet transform," IEEE Trans. Image Proc., 2003. submitted, http://www.ifp.uiuc.edu/ minhdo/publications. 